\author{
Conservación
}

\title{
Modelación de la distribución del oso andino Tremarctos ornatus en el bosque seco del Marañón (Perú)
}

\author{
Modeling of Andean bear Tremarctos ornatus distribution in the dry forest of Marañón (Peru) \\ Judith Figueroa $^{\mathrm{a}, \mathrm{b}, *}$, Marcelo Stucchi ${ }^{\mathrm{a}}$ y Roxana Rojas-VeraPinto ${ }^{\mathrm{c}, \mathrm{d}}$ \\ ${ }^{a}$ Asociación para la Investigación y Conservación de la Biodiversidad, Av. Vicús 538, Lima 33, Perú \\ ${ }^{\mathrm{b}}$ Estación Biológica Terra Natura, Grupo de Investigación Zoología de Vertebrados, Instituto Universitario de Investigación, Centro Iberoamericano de la \\ Biodiversidad, Universidad de Alicante, Apartado 99, E-03080, Alicante, España \\ c Proyecto Isnachi, Calle 5 manzana D-lote 16, Urbanización La Libertad, Lima 33, Perú \\ ${ }^{\mathrm{d}}$ Frankfurt Zoological Society-Perú, Calle Los Cipreses H-21, Residencial Huancaro, Santiago, Cusco, Perú \\ Recibido el 17 de marzo de 2015; aceptado el 8 de septiembre de 2015 \\ Disponible en Internet el 18 de febrero de 2016
}

\begin{abstract}
Resumen
El oso andino (Tremarctos ornatus) es una especie en situación vulnerable, cuyo hábitat se va fragmentando poco a poco por la expansión de las actividades humanas. En el Perú, el bosque seco del Marañón, ecorregión poco investigada a nivel biológico y ecológico, es también hábitat de la especie. Lamentablemente, en la actualidad está siendo afectada por la agricultura, ganadería y construcción de vías de comunicación, y en el futuro cercano se verá amenazada por la implementación de actividades económicas de gran envergadura, como las centrales hidroeléctricas. Con el objetivo de identificar áreas prioritarias para la investigación y conservación del oso andino en el bosque seco del Marañón de las regiones Cajamarca y Amazonas, se modeló su hábitat utilizando el algoritmo MaxEnt. Se emplearon 60 registros de presencia, así como 6 variables ambientales. De un total de $6,501.2 \mathrm{~km}^{2}$ de bosque seco del Marañón, el hábitat adecuado para el oso andino sería solo de 480.7 km² $(7.4 \%)$ y el hábitat marginal de $2,418.5 \mathrm{~km}^{2}(37.2 \%)$. Se identificaron 3 sectores prioritarios de investigación y conservación que presentan, además, conectividad con bosques montanos y páramos de áreas protegidas: Parque Nacional de Cutervo, Área de Conservación Municipal Huangamarca (Cajamarca), Áreas de Conservación Privada Huiquilla y Huaylla Belén-Colcamar (Amazonas).

Derechos Reservados (C) 2015 Universidad Nacional Autónoma de México, Instituto de Biología. Este es un artículo de acceso abierto distribuido bajo los términos de la Licencia Creative Commons CC BY-NC-ND 4.0.
\end{abstract}

Palabras clave: Amazonas; Áreas protegidas; Bosque seco del Marañón; Cajamarca; Conectividad; Conservación; Oso andino; MaxEnt

\begin{abstract}
The Andean bear (Tremarctos ornatus) is a vulnerable species, his habitat is gradually fragmented by the expansion of human activities. In Peru, the Marañón dry forest, an ecoregion with little biological and ecological research, is the habitat of the species. Unfortunately, at the present time, it is being affected by agriculture, livestock and road construction, and soon will be threatened by the implementation of large scale economic activities, such as hydroelectric stations. In order to identify priority sectors for research and conservation of the Andean bear in the Marañón dry forest of Cajamarca and Amazonas regions, its habitat was modeled using MaxEnt algorithm. Sixty records of its presence as well as 6 environmental and climatic variables were used. Considering a total extension of $6,501.2 \mathrm{~km}^{2}$ of Marañon dry forest, the suitable existing habitat for the Andean bear would be only $480.7 \mathrm{~km}^{2}(7.4 \%)$ and the marginal existing habitat, $2,418.5 \mathrm{~km}^{2}(37.2 \%)$. Three priority sectors for research and conservation were identified which also present connectivity to montane forests and paramos inside protected areas: Cutervo National Park, Huangamarca Municipal Conservation Area (Cajamarca) and Private Conservation Areas, Huiquilla and Huaylla Belén-Colcamar (Amazonas). All Rights Reserved @ 2015 Universidad Nacional Autónoma de México, Instituto de Biología. This is an open access item distributed under the Creative Commons CC License BY-NC-ND 4.0.
\end{abstract}

Keywords: Amazonas; Protected areas; Dry forest of Marañón; Cajamarca; Connectivity; Conservation; Andean bear; MaxEnt

\footnotetext{
* Autora para correspondencia.

Correo electrónico: aicb.peru@gmail.com (J. Figueroa).

La revisión por pares es responsabilidad de la Universidad Nacional Autónoma de México.
} 


\section{Introducción}

El bosque seco del Marañón (BSM) es un ecosistema xérico que presenta un alto grado de diversidad y endemismo, en comparación con otros bosques con similares características ecológicas, como el bosque seco ecuatorial y el bosque seco interandino (Linares-Palomino y Pennington, 2007). Se ubica en el norte del Perú, abarcando parte de las regiones Lambayeque, Cajamarca, Amazonas, La Libertad y Ancash (Olson et al., 2001). Su grado de endemismo se debe a su aislamiento geográfico, ya que se encuentra rodeado de matorrales y bosques montanos húmedos y páramos, en las partes más altas (Killeen, Douglas, Consiglio, Jørgensen y Mejía, 2007). Asimismo, presenta especies relacionadas con el bosque seco de la franja costera del Pacífico y con la Amazonía (Brack y Mendiola, 2004). A pesar de su valor ecológico, esta es una de las regiones naturales menos exploradas en el Perú, que además no se encuentra protegida por la administración nacional representada por el Sistema Nacional de Áreas Naturales Protegidas por el Estado (Sernanp, 2014). En la actualidad, el BSM está siendo rápidamente afectado y fragmentado por diversas actividades antrópicas como la agricultura, ganadería y construcción de vías de comunicación, lo que ha originado la pérdida del $48.4 \%$ del bosque seco en esas regiones (Figueroa, Stucchi y RojasVeraPinto, 2013).

Una de las especies más sobresalientes de esta ecorregión es el oso andino (Tremarctos ornatus), el cual se encuentra en situación vulnerable a nivel global (IUCN, 2014) y en el Perú (Minagri, 2014). La colonización humana de las áreas boscosas para el desarrollo de actividades agropecuarias es una de las causas que contribuye al declive poblacional de la especie, debido a que propicia la cacería y la fragmentación de su hábitat (Rodríguez et al., 2003), por lo que la pérdida acelerada del BSM estaría afectando negativamente a las poblaciones que se desplazan o habitan dentro de él.

La falta del conocimiento detallado de la distribución de las especies amenazadas, poco conocidas o invasoras, inclusive en escenarios históricos o futuros, representa una seria preocupación en la gestión y conservación de la fauna silvestre, siendo esta una de las prioridades para cualquier toma de decisiones y planes de acción. Es así que la modelación del hábitat es una de las herramientas más utilizadas en la actualidad para mejorar este conocimiento, permitiendo revelar información sobre los requerimientos ambientales de estos (Nazeri, Jusoff, Madani, Mahmud y Bahman, 2012). Las modelaciones que requieren solo datos de presencia y variables ambientales son las más utilizadas debido a la mayor accesibilidad de información, siendo las más conocidas el algoritmo genético para la predicción de un conjunto de reglas (GARP), el análisis factorial del nicho ecológico (ENFA) y la máxima entropía (MaxEnt). Esta última es ampliamente utilizada ya que, por lo general, tiene un mejor desempeño que los otros modelos (Elith et al., 2006; Pearson, Raxworthy, Nakamura y Peterson, 2007). Asimismo, tiene la capacidad de ser poco sensible al tamaño de la muestra utilizada para la predicción (Wisz et al., 2008).

MaxEnt estima la probabilidad de distribución de una especie encontrando su máxima entropía, es decir, lo más dispersa o cercana a la uniformidad posible, pero sujeta a un conjunto de restricciones que representan la información incompleta que se tiene sobre esta (Phillips, Anderson y Schapire, 2006). En este sentido, el objetivo del presente estudio fue identificar las áreas donde potencialmente se distribuye el oso andino en el BSM utilizando el principio de máxima entropía, para que sirva como un primer paso para futuras evaluaciones en el campo que nos permitan plantear estrategias que contribuyan a la conservación de la especie.

\section{Materiales y métodos}

Se incluyó el BSM de las regiones Cajamarca y Amazonas, entre las cordilleras occidental y oriental de los Andes. Figueroa et al. (2013) calcularon que tuvo una cobertura histórica de $12,588.6 \mathrm{~km}^{2}$ y que, debido a la deforestación, actualmente comprende un área de $6,501.2 \mathrm{~km}^{2}$ (fig. 1). Se caracteriza por tener un clima cálido; con una temperatura media anual entre 23 y $24^{\circ} \mathrm{C}$, que puede superar los $40^{\circ} \mathrm{C}$ en los valles más bajos del río Marañón. Tiene escasas precipitaciones, entre 100 y $500 \mathrm{~mm}$, que se producen solo en los meses de verano, entre diciembre y marzo (Brack y Mendiola, 2004). Se reconocen 3 asociaciones botánicas: $a$ ) asociación de especies subespinosas y subxerófitas, la cual representa el ecotono entre el matorral mesotérmico de ladera media y el bosque seco, que se distribuye entre los 2,530 y los $1,650 \mathrm{~m} \mathrm{snm} ; b$ ) asociación de caducifolios y espinosos, entre los 1,650 y los 1,550 $\mathrm{m}$ snm y $c$ ) asociación de xerófitos pluvifolios con cactáceas columnares, entre los 1,550 y los 900 m snm (Sánchez-Vega y Sánchez-Rojas, 2012).

\section{Registros espaciales de la especie}

Se utilizaron registros georreferenciados (latitud/longitud) de observación de osos o rastros de estos, obtenidos a través de 3 fuentes: $a$ ) información dentro del BSM: se realizaron entrevistas semiestructuradas a 69 pobladores de 21 comunidades presentes en los bosques secos y montanos adyacentes al río Marañón, dentro del sector Jaén y Balsas $\left(5^{\circ} 42^{\prime} \mathrm{S}, 78^{\circ} 40^{\prime} \mathrm{O}\right.$ y $6^{\circ} 50^{\prime} \mathrm{S} ; 7^{\circ} 01^{\prime} \mathrm{O}$ ) (fig. 1); los pobladores fueron principalmente cazadores, agricultores y ganaderos, entre 23 y 95 años de edad; algunos de los puntos fueron visitados y georreferenciados; $b$ ) información regional: debido a la escasa información georreferenciada sobre el oso andino en el BSM, se procedió a la búsqueda de datos de la especie en otros hábitats de Cajamarca y Amazonas; se revisaron 31 informes y artículos publicados; c) también se obtuvo por medio de los registros de otros investigadores mediante comunicaciones personales.

Todos los puntos de presencia pasaron por un proceso de selección para evitar la intensidad de sobremuestreo y dependencia. Para esto, los registros elegidos debían tener una distancia mínima de zona buffer de $3 \mathrm{~km}$ entre ellos, obteniendo así 60 puntos (fig. 2a). De ellos, para evaluar el modelo con receiver operating characteristic curve (ROC) parcial, se seleccionaron 18 puntos al azar (30\%).

Para la elección de las variables se eligieron los datos ambientales proporcionados por WorldClim, versión 1.4 (www.worldclim.org; Hijmans, Cameron, Parra, Jones y Jarvis, 


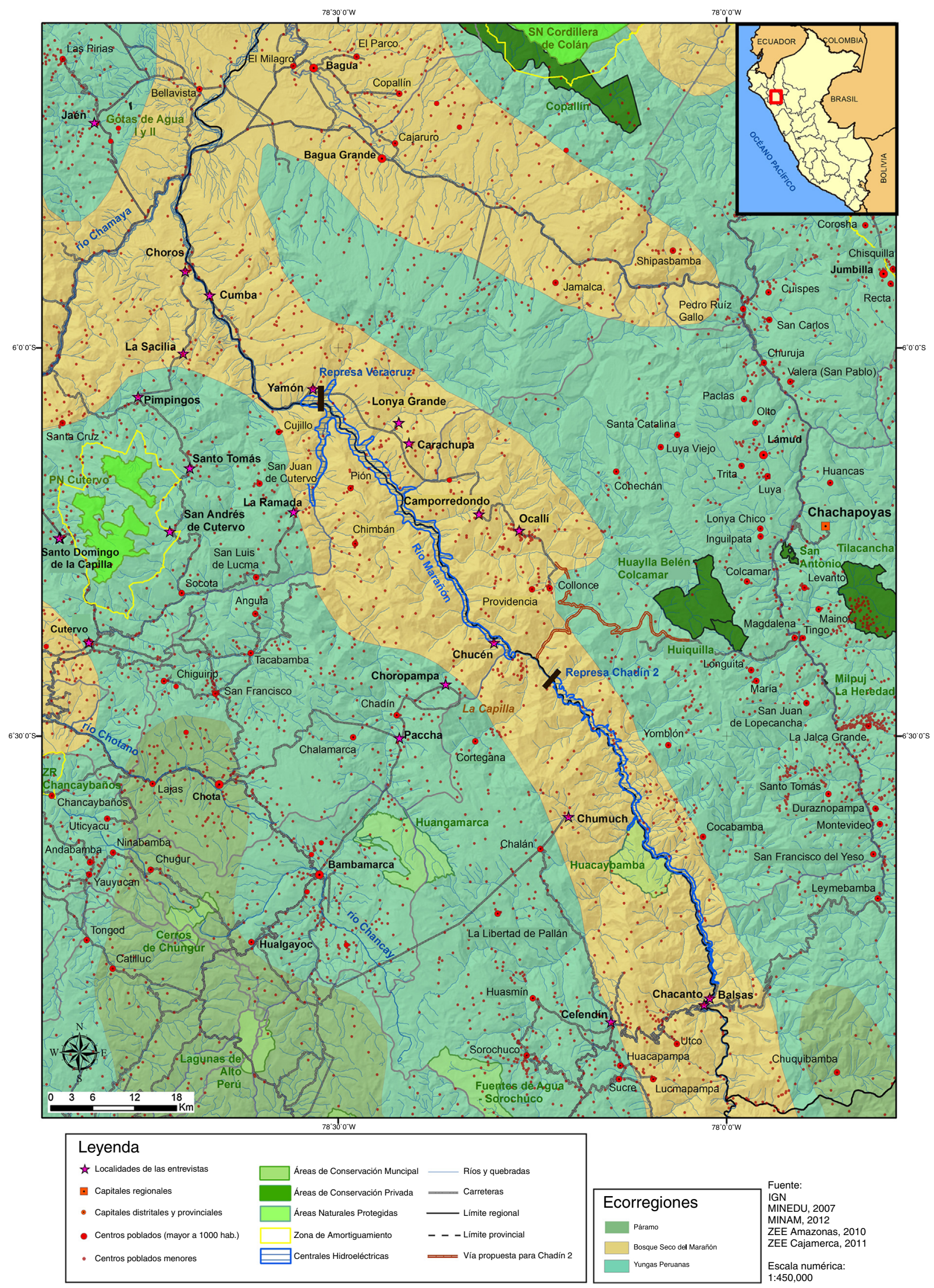

Figura 1. Localización geográfica del bosque seco del Marañón (Cajamarca-Amazonas). 


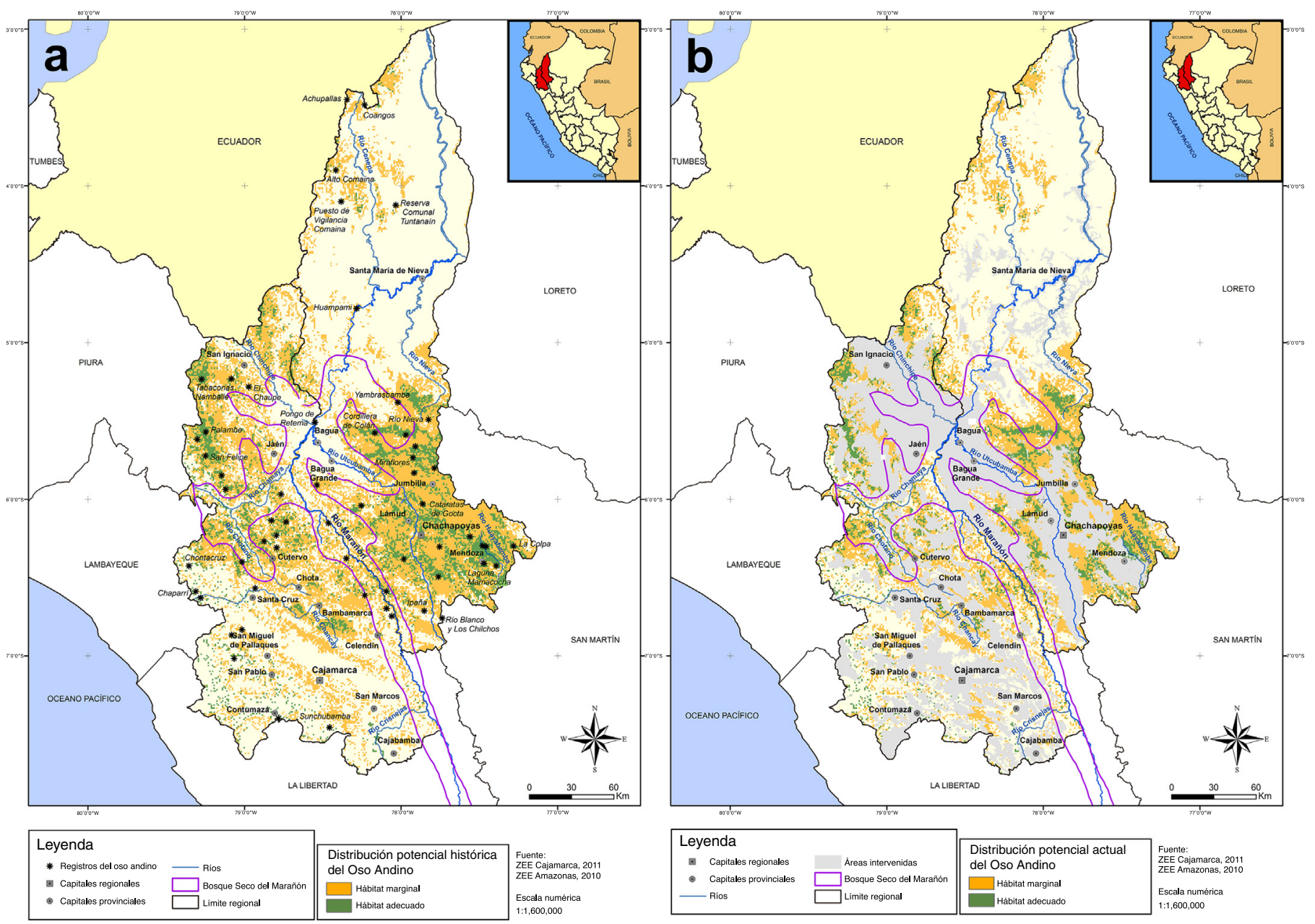

Figura 2. Modelación de la distribución del oso andino en las regiones Cajamarca y Amazonas: a) distribución potencial histórica; b) distribución potencial actual.

$2005)$, que no fueran altamente correlacionadas $(\mathrm{r}<0.75)$ y que contribuyan a la predicción de la distribución potencial de la especie. Es así que después de efectuar varias corridas del programa, se seleccionaron 6 variables ambientales: intervalo de temperatura diurna (bio 2), isotermalidad (bio 3), intervalo de temperatura anual (bio 7), temperatura media del trimestre más seco (bio 9), precipitación del mes más seco (bio 14) y estacionalidad de precipitación (bio 15). Además, en estudios previos sobre el uso del hábitat por parte del oso andino, se encontró que la altitud y la pendiente son variables asociadas a su presencia (Cuesta, Peralvo y van Manen, 2003), por lo que también se incluyeron con el fin de conocer su desempeño en la modelación. Ambas fueron obtenidas del consortium for spatial information (CGIAR-CSI, 2013) (tabla 1).

\section{Datos antrópicos}

Con el fin de conocer la distribución potencial actual del oso andino e identificar las zonas de mayor impacto, se utilizó el programa ArcGis, versión 10.1 para superponer los datos antrópicos sobre el mapa obtenido en la modelación. La información georreferenciada fue proporcionada por los gobiernos regionales de Cajamarca y Amazonas, de sus zonificaciones ecológicas económicas (GR-Amazonas e IIAP, 2010; GR-Cajamarca, 2011).
Tabla 1

Variables usadas y sus aportes en el modelamiento del hábitat del oso andino en Cajamarca y Amazonas, Perú.

\begin{tabular}{lll}
\hline Código & Descripción & Aporte al modelo \\
\hline Bio 2 & $\begin{array}{l}\text { Intervalo de temperatura } \\
\text { diurna (media mensual } \\
\text { (temperatura } \\
\text { máxima-temperatura } \\
\text { mínima)) }\end{array}$ & 0.2 \\
Bio 3 & $\begin{array}{l}\text { Isotermalidad (bio 2/bio 7) } \\
\text { (100) }\end{array}$ & 15.7 \\
Bio 7 & $\begin{array}{l}\text { Intervalo de temperatura } \\
\text { anual }\end{array}$ & 4.1 \\
Bio 9 & $\begin{array}{l}\text { Temperatura media del } \\
\text { trimestre más seco }\end{array}$ & 10.2 \\
Bio 14 & $\begin{array}{l}\text { Precipitación del mes más } \\
\text { seco }\end{array}$ & 7.6 \\
Bio 15 & $\begin{array}{l}\text { Estacionalidad de } \\
\text { precipitación }\end{array}$ & 61.7 \\
Altitud & $\begin{array}{l}\text { Modelo digital del terreno } \\
\text { (MDT) que muestra la } \\
\text { elevación de la superficie } \\
\text { Grado de inclinación de la } \\
\text { superficie }\end{array}$ \\
\hline Pendiente &
\end{tabular}


Tabla 2

Hábitat adecuado y marginal del oso andino en el bosque seco del Maranón (Cajamarca y Amazonas).

\begin{tabular}{|c|c|c|c|}
\hline Bosque seco del Marañón & Extensión $\left(\mathrm{km}^{2}\right)$ & $\%$ cobertura histórica & $\%$ cobertura actual \\
\hline Cobertura histórica & $12,588.6$ & & \\
\hline Cobertura actual & $6,501.2$ & & \\
\hline Hábitat marginal histórico & $4,171.5$ & 33.1 & \\
\hline Hábitat adecuado actual & 480.7 & 3.8 & 7.4 \\
\hline Hábitat marginal actual & $2,418.5$ & 19.2 & 37.2 \\
\hline Cobertura histórica & $6,892.1$ & & \\
\hline Cobertura actual & $3,917.2$ & & \\
\hline Hábitat adecuado histórico & 467.9 & 3.7 & \\
\hline Hábitat marginal histórico & $2,428.0$ & 19.3 & \\
\hline Hábitat adecuado actual & 304.7 & 2.4 & 4.7 \\
\hline Hábitat marginal actual & $1,450.2$ & 11.5 & 22.3 \\
\hline Hábitat marginal histórico & $1,743.5$ & 13.8 & \\
\hline Hábitat adecuado actual & 176.0 & 1.4 & 2.7 \\
\hline Hábitat marginal actual & 968.3 & 7.7 & 14.9 \\
\hline
\end{tabular}

La zonificación ecológica económica es un instrumento que permite identificar las diferentes alternativas de uso sostenible en las regiones, basadas en la evaluación de sus potencialidades y limitaciones con criterios físicos, biológicos, sociales, económicos y culturales. Dentro del aspecto antrópico, se incluyeron los efectos de las actividades humanas sobre la tierra, tales como patrones de asentamientos humanos, usos del suelo, explotaciones económicas y ocupación sociocultural de las zonas ecológicas (Rodríguez-Achung, 2007). La información espacial sobre la red vial nacional se obtuvo del Ministerio de Transporte y Comunicaciones (MTC, 2013) y la de los centros poblados, del Censo Poblacional de 2007 (INEI, 2007). El mapa con la modelación del hábitat del oso andino sin influencia antrópica se denominó «distribución potencial histórica» (fig. 2a), mientras que el mapa con influencia antrópica se llamó «distribución potencial actual» (fig. 2b). El cálculo del área de ambas distribuciones y los datos de deforestación y cobertura del BSM en Cajamarca y Amazonas (Figueroa et al., 2013) nos permitió estimar la pérdida del hábitat del oso andino.

\section{Desarrollo del modelo}

Se aplicó el algoritmo MaxEnt, versión 3.3.3 k (http://www. cs.princeton.edu/ schapire/maxent/; Phillips et al., 2006). Para la edición de las capas ambientales se utilizó el programa ArcGis, versión 10.1; este permitió recortar y transformar las capas ambientales seleccionadas, con el fin de obtener la misma extensión, el mismo tamaño de pixel (aproximadamente $1 \mathrm{~km}^{2}$ ) y la misma posición de estos. Antes de ejecutar el modelo se procedió a darle algunos ajustes previos al proceso, el resto se dejó por defecto: $a$ ) formato de salida: acumulativo (rango 0 al 100), ya que indica la idoneidad del hábitat relativo de cada pixel (Benítez, 2010); b) tipo de capas: lineal y cuadrática, indican las condiciones promedio de presencia, tolerancia de la especie a la variación y correlación de las variables (Delgado, 2008); c) tipo de réplicas a correr: bootstrap (Pearson, 2007).

Frente a las limitaciones que presenta la evaluación del área debajo de la curva (Lobo, Jiménez-Valverde y Raimundo, 2008), se optó por trabajar con el ROC parcial para evaluar de forma extrínseca el modelo y su habilidad de predicción (Peterson, Papeş y Soberón, 2008). Se calculó este valor usando la herramienta Partial-ROC, versión 1.0 (Barve, 2008), previo ajuste: $a$ ) proporción de puntos en bootstrap: $75 ; b$ ) remuestreo con reemplazo: 1,$000 ; c) 1$-umbral de omisión: para elegir el umbral y así transformar el mapa a binario (presencia-ausencia), se determinó un error de omisión aceptable - deja fuera puntos de presencia de la especie, ya que si bien pasó por allí, no es realmente un hábitat adecuado para este - con base en la experiencia en campo. Es así que después de evaluar diferentes umbrales se estableció que el valor 0.88 (error de omisión de $12 \%$ ) era el más adecuado y aceptable para el contexto del presente trabajo frente al de $10 \%$ (utilizado por Nazeri et al., 2012) o la elección de alguna regla de umbral establecido por el MaxEnt.

\section{Resultados}

El valor del desempeño del modelo con un error de omisión de $12 \%$ tuvo una puntuación de área debajo de la curva ratio de $1.51 \pm 0.09$ y una omisión baja $(\mathrm{p}<0.001)$. Esto significa que el umbral elegido para la salida acumulativa del modelo descartó los valores de 0-19 como hábitat idóneo para el oso andino. Posteriormente, se clasificó el mapa resultante en hábitat marginal (20-59\%) y hábitat adecuado (60-100\%). El porcentaje de contribución de cada variable ambiental se obtuvo por medio del análisis Jacknife, siendo la variable de estacionalidad de precipitación la que más aportó (61.7\%), seguida de isotermalidad 
(15.7\%) y temperatura media del trimestre más seco (10.2\%). Es interesante resaltar que para la zona de estudio la variable pendiente no tuvo ninguna contribución para el modelo $(0 \%)$ (tabla 1); esto puede deberse a que los osos no se ven limitados por terrenos con pendientes muy pronunciadas, donde incluso se alimentan, como se ve normalmente en el bosque seco ecuatorial de Lambayeque, norte del Perú (Figueroa y Stucchi, 2009).

Siendo el área actual del BSM en Cajamarca y Amazonas de $6,501.2 \mathrm{~km}^{2}$, el hábitat adecuado para oso andino sería de $480.7 \mathrm{~km}^{2}$ (7.4\%) y marginal de $2,418.5 \mathrm{~km}^{2}(37.2 \%)$; con estos valores se podría inferir que el 55.4\% corresponde a un hábitat inadecuado para la especie. Cajamarca presenta en la actualidad $3,917.2 \mathrm{~km}^{2}$ de BSM, en donde el hábitat adecuado actual del oso andino es de $304.7 \mathrm{~km}^{2}$ (4.7\%) y el hábitat marginal de $1,450.2 \mathrm{~km}^{2}$ (22.3\%). En Amazonas, el área del BSM es menor con $2,584.0 \mathrm{~km}^{2}$, en donde el hábitat adecuado actual del oso andino es de $176.0 \mathrm{~km}^{2}$ (2.7\%) y el hábitat marginal de $968.3 \mathrm{~km}^{2}$ (14.9\%) (tabla 2).

$\mathrm{Al}$ sobreponer las actividades antrópicas se reconocen las zonas con hábitat adecuado y marginal actual para el oso andino. Es así que en el mapa de distribución (fig. 3), en el norte de Cajamarca, se observa un parche de hábitat marginal del oso en los alrededores de San Pedro. En la parte media, observamos pequeños parches dispersos de hábitat marginal en los alrededores del río Silaco y Chimbán, Pedernal, Las Palmas, Tapón y Chucén. Entre Cortegana y Chumuch encontramos parches más amplios de hábitat adecuado, con una importante presencia de hábitat marginal en los alrededores del área de conservación municipal Huacaybamba-Cerro Cedropata, Tolón y Shucamayo. En el BSM de Amazonas, en los alrededores de Shipasbamba se observan parches de hábitat marginal y adecuado para el oso andino. En la parte media, en los alrededores de Pueblo Viejo hay un importante parche de hábitat adecuado. Hacia el sur, entre Huarapo hasta la altura de Tuén se encuentran parches continuos de hábitat marginal, con hábitat adecuado en los alrededores de Yomblón.

\section{Discusión}

\section{Pérdida del hábitat del oso andino}

Aproximadamente el 50\% de la cobertura boscosa del BSM en Cajamarca y Amazonas se ha perdido, principalmente en los últimos 50 años, debido a diversas actividades antrópicas (Figueroa et al., 2013). Esta pérdida también incluye los hábitats adecuado y marginal del oso andino (tabla 2), los cuales han sido destinados a la agricultura, ganadería y minería. En Amazonas existe una carretera paralela al río Marañón que va desde Corral Quemado hasta Collonce, que pasa por Cumba y que será ampliada hasta Cohechán para unirse a la vía que va hacia Luya, y de ahí a Chachapoyas — capital de la región Amazonas-. Esta carretera atravesará el corredor propuesto como área prioritaria de conservación Vilaya-Condorpuna (Romo, Leo y Epiquién, 2009), que presenta una importante área de hábitat adecuado para el oso andino. Al igual que en el resto de los proyectos viales en el Perú, no se ha tomado en cuenta la implementación de pasos de fauna. Asimismo, existe una vía que une Chachapoyas con Tingo, cuya prolongación hasta la altura de Saquilillo será la vía de acceso para la construcción de la represa de la central hidroeléctrica Chadín 2 (Amec-Perú, 2011), la cual ocasionará diversos impactos antrópicos en el área, al igual que la represa de la hidroeléctrica Veracruz (OY Ingeniería, 2009), cerca de la confluencia de los ríos Silaco y Marañón (Figueroa et al., 2013) (fig. 1).

La construcción de la carretera Corral Quemado-Lonya Grande se inició en la década de 1960, lo que facilitó el ingreso de los cazadores y madereros a este sector, así como al cerro Condorpuna. En la actualidad, esta carretera permite la salida de café y frutales desde Ocallí, Camporredondo, Lonya Grande y Yamón (Amazonas) hacia Jaén (Cajamarca) y Chiclayo, capital de la región Lambayeque, así como el ingreso de pobladores de otras regiones que llegan a la zona en busca de tierras de cultivo, lo que ha influido en la rapidez de la fragmentación de los bosques secos de este sector. En Cajamarca, desde Choropampa hacia el Marañón también se observó un gran impacto en el bosque debido a la fragmentación por ganadería y cultivos, la cual fue acelerada por las carreteras Chadín-Choropampa y Chadín-La Capilla-Choropampa. De igual forma, se prevé la continuación de la carretera Choropampa-Chucén, que permitirá la salida hacia el río Marañón, lo cual impactará directamente en el bosque seco (fig. 1).

Además de la pérdida del hábitat del oso andino, todas las personas entrevistadas señalaron que dentro del BSM existen algunos conflictos entre los pobladores con la especie, debido a su ingreso a los campos de cultivo para alimentarse de maíz (Zea mays), plátano (Musa paradisiaca), caña de azúcar (Saccharum officinarum) y yuca (Manihot esculenta); depredación de ganado vacuno, uso de las partes del oso como alimento y en la medicina tradicional, así como la comercialización de individuos y partes del oso. Estos mismos conflictos se repiten en otras áreas de Cajamarca y Amazonas, los cuales en la mayoría de los casos concluyen con la cacería de la especie (Butchart, Barnes, Davies, Fernández y Seddon, 1995; Peyton, 1980; Vela, 2009).

\section{Áreas prioritarias para la investigación y conservación del oso andino}

La figura 3 muestra que el hábitat marginal y adecuado del oso andino está fragmentado prácticamente en todo el BSM, y esto podría suponer una grave amenaza para la supervivencia a largo plazo de la especie. Por ello, queda claro que, para su conservación, los planteamientos deben enfocarse desde una perspectiva integral de los ecosistemas, mantener un amplio intervalo altitudinal y no a través de áreas protegidas pequeñas y aisladas (Bennet, 1998; Yerena, 1993). En el caso del BSM, a pesar de los impactos del área, aún se identificaron algunas zonas en donde se mantiene un paisaje que podría presentar una alta conectividad, en donde los osos se desplazarían entre el bosque seco, el bosque montano y el páramo para alimentarse, protegerse y reproducirse. Incluso, el río Marañón parece no representar una barrera física para el oso andino, principalmente en la época de estiaje, ya que en las entrevistas del presente estudio lo señalan como un buen nadador. Este comportamiento también fue registrado en Ecuador (Castellanos, Altamirano y Tapia, 2005). 


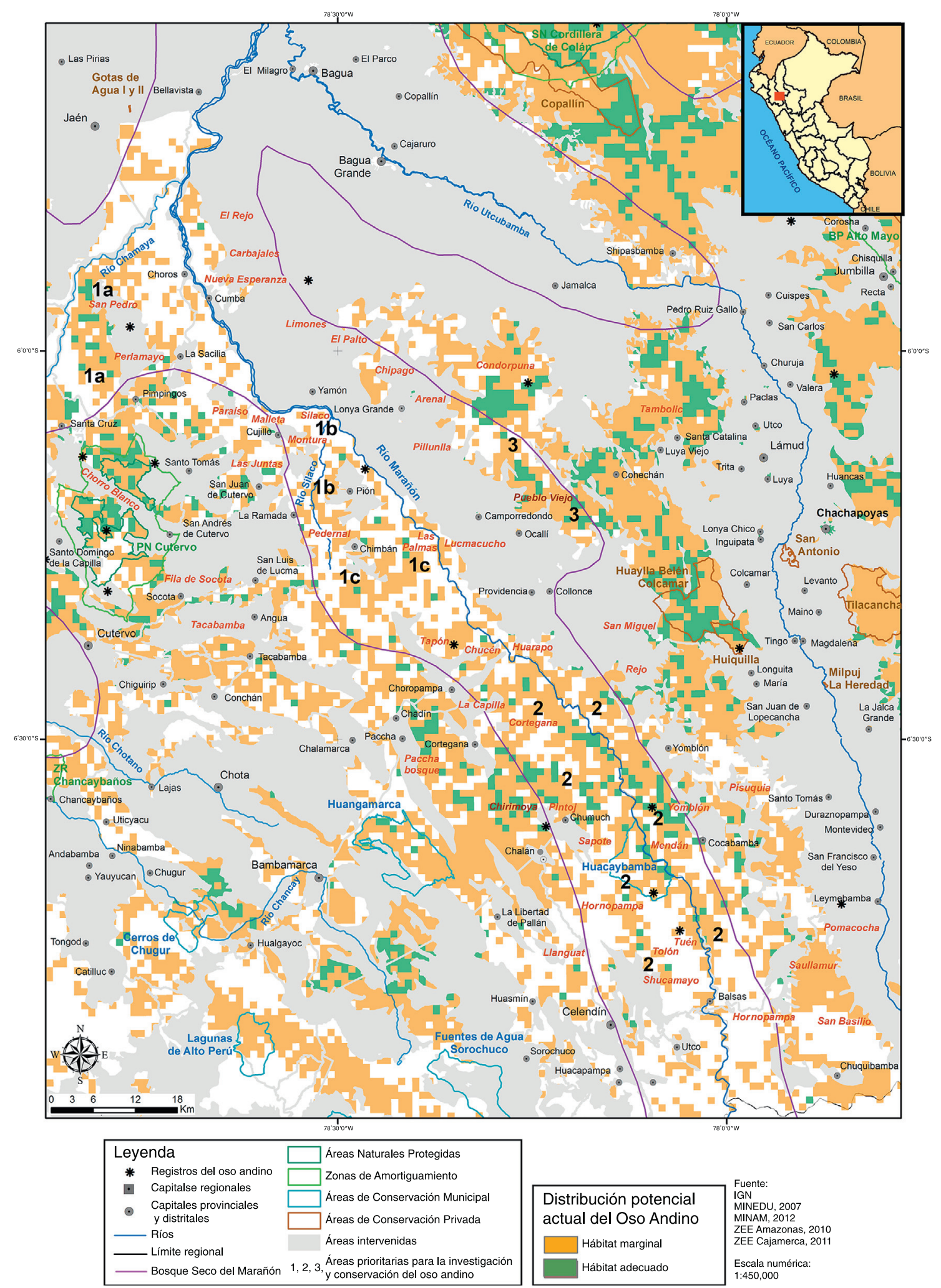

Figura 3. Modelación de la distribución del oso andino en el bosque seco del Marañón (Cajamarca-Amazonas): Área prioritaria de conservación e investigación 1, incluye los 3 ramales de hábitats marginales dentro del BSM conectados con los hábitats adecuados y marginales dentro del Parque Nacional de Cutervo y su zona de amortiguamiento (ZA). Dentro de esta existen 3 subáreas: a) los bosques montanos al norte del parque y su ZA con el BSM de los alrededores de San Pedro y Perlamayo; b) los bosques montanos y páramos ubicados en el sur del bloque norte del parque y su ZA, San Andrés de Cutervo y San Juan de Cutervo, con el BSM de los alrededores del río Silaco; y c) el bloque sur del parque y su ZA, Sócota y San Luis de Lucma, con el BSM de los alrededores de Chimbán y Las Palmas. Área prioritaria de conservación e investigación 2, incluye el BSM de los bosques de Chumuch-Cortegana, el Área de Conservación Municipal Huacaybamba-Cerro Cedropata, Tolón y Shucamayo. Área prioritaria de conservación e investigación 3, incluye el BSM de los alrededores de Pueblo Viejo. 
En Cajamarca existen 2 áreas prioritarias para la conservación e investigación: la primera incluiría los 3 ramales de hábitats marginales dentro del BSM conectados con los hábitats adecuados y marginales dentro del Parque Nacional de Cutervo y su zona de amortiguamiento (ZA): $a$ ) los bosques montanos al norte del parque y su ZA con el BSM de los alrededores de San Pedro y Perlamayo (fig. 3-1a); b) los bosques montanos y páramos ubicados en el sur del bloque norte del parque y su ZA, San Andrés de Cutervo y San Juan de Cutervo, con el BSM de los alrededores del río Silaco (fig. 3-1b) y c) el bloque sur del parque y su ZA, Sócota y San Luis de Lucma, con el BSM de los alrededores de Chimbán y Las Palmas (fig. 3-1c). La segunda área prioritaria (fig. 3-2) incluiría el BSM de los bosques de Chumuch-Cortegana (GR-Cajamarca, 2011), Área de Conservación Municipal Huacaybamba-Cerro Cedropata (Roncal-Rabanal, Díaz-Mori, Roncal-Alcántara y Rabanal-Díaz, 2013), Tolón y Shucamayo. Toda esta área se encuentra conectada hacia el oeste con los bosques montanos de Chalán y del Área de Conservación Municipal Huangamarca. Asimismo, el área descrita también presenta conexión hacia el este, en la región Amazonas. Esta se conecta con el BSM de Tuén, Mendán y Yomblón, que a su vez tiene continuidad con bosques montanos y páramos ubicados al noreste siguiendo de forma paralela al río Marañón. Por el norte con Pisuquia, áreas de conservación privada Huiquilla y Huaylla Belén-Colcamar y Corredor Vilaya-Condorpuna. Este gran bloque se conecta con los BSM de los alrededores de Pueblo Viejo (fig. 3-3). Hacia el sur observamos continuidad con los bosques montanos de Saullamur y San Basilio, donde se tienen registros del oso en la actualidad.

Esta primera etapa nos delimita el área de trabajo prácticamente a los 3 sectores descritos. Si bien los modelos de distribución potencial de las especies permiten realizar inferencias sobre estas, presentan sus propias limitaciones metodológicas, por lo que será importante realizar una evaluación in situ para verificar la presencia actual del oso andino en dichas zonas y sustentar su consideración como áreas prioritarias para la conservación de la especie dentro del BSM. Es importante resaltar que las propuestas de áreas prioritarias para la conservación e investigación deben agotar todos los esfuerzos de estudio, incluyendo los de conectividad, con el fin de que estén bien fundamentadas para que su implementación sea a corto plazo.

\section{Agradecimientos}

Queremos expresar nuestro agradecimiento a M. Mindreau por su iniciativa para la realización del presente estudio y su apoyo en el desarrollo del mismo. A O. Vílchez, N. Shanee, M. Enciso, M. Roncal, F. Roca, L. Troyes, P. Heredia, H. Plenge, P. Cabrera, F. Angulo-Pratolongo, M. Aldave, J. Ramos, P. Calvay, I. Zárate, C. Segura, J. Gutiérrez, E. Fernández por su ayuda con información sobre la zona. A los pobladores de las comunidades visitadas, especialmente a R. Vallejos, A. Díaz, F. Ludeña, V. Salazar, B. Guevara, H. Soto, S. Soto, L. Pérez, E. Figueroa, R. Herrera, A. Valdivia, M. Mori, I. Escobedo, L. Vela y J. F. Salcedo por su ayuda en el trabajo de campo. A E. Terán, P.
Velazco, D. Gálvez-Roeder, R. Pinedo y R. Linares-Palomino por las referencias bibliográficas brindadas. A la Cooperación Alemana-GIZ por el financiamiento de la investigación. A P. Atauchi-Rojas por sus valiosas sugerencias en la modelación, así como a los 3 revisores anónimos por sus acertados comentarios en la revisión del manuscrito.

\section{Referencias}

Amec-Perú. (2011). Estudio de impacto ambiental proyecto central hidroeléctrica Chadín 2. Lima, Perú: Regiones Amazonas y Cajamarca, A.C., Energía, S.A., Odebrecht Energía.

Barve, N. (2008). Tool for partial-ROC. Lawrence, Kansas: Biodiversity Institute. Versión 1.0

Benítez, A. (2010). Aproximaciones del hábitat potencial para jaguar (Panthera onca) en la región Caribe colombiana. Tesis de maestría. Centro Agronómico Tropical de Investigación y Enseñanza.

Bennet, A. F. (1998). Linkages in the landscape: the role of corridors and connectivity in wildlife conservation. Gland, Suiza y Cambridge, Reino Unido: IUCN.

Brack, A. y Mendiola, C. (2004). Ecología del Perú. Lima, Perú: Programa de las Naciones Unidas para el Desarrollo.

Butchart, S., Barnés, R., Davies, C., Fernández, M. y Seddon, N. (1995). Threatened mammals of the cordillera de Colán, Peru. Oryx, 29, 275-281.

Castellanos, A., Altamirano, M. y Tapia, G. (2005). Ecología y comportamiento de osos andinos reintroducidos en la Reserva Biológica Maquipucuna. Ecuador: implicaciones en la conservación. Revista Politécnica, 26, 54-82.

CGIAR-CSI (Consultative Group for International Agricultural ResearchConsortium for Spatial Information). (2013). Recuperado el 25 de noviembre de 2013 de: www.cgiar-csi.org.

Cuesta, F., Peralvo, M. F. y van Manen, F. T. (2003). Andean bear habitat use in the Oyacachi River Basin, Ecuador. Ursus, 14, 198-209.

Delgado, T. (2008). Evolución de la diversidad vegetal en Ecuador ante un escenario de cambio global. Tesis doctoctoral. Universidad Complutense de Madrid.

Elith, J., Graham, C. H., Anderson, R. P., Dudik, M., Ferrier, S., Guisan, A., et al. (2006). Novel methods improve prediction of species' distributions from occurrence data. Ecography, 29, 129-151.

Figueroa, J. y Stucchi, M. (2009). El oso andino: alcances sobre su historia natural. Lima, Perú: Asociación para la Investigación y Conservación de la Biodiversidad.

Figueroa, J., Stucchi, M. y Rojas-VeraPinto, R. (2013). El oso andino (Tremarctos ornatus) como especie clave para la conservación del bosque seco del Marañón (Cajamarca-Amazonas, Perú). Lima, Perú: Cooperación Técnica Alemana, Asociación para la Investigación y Conservación de la Biodiversidad.

GR-Amazonas e IIAP (Gobierno Regional de Amazonas e Instituto de Investigación de la Amazonía Peruana). (2010). Proyecto zonificación ecológica y económica del departamento de Amazonas. Iquitos, Perú: Gobierno Regional de Amazonas e Instituto de Investigación de la Amazonía Peruana.

GR-Cajamarca (Gobierno Regional de Cajamarca). (2011). Zonificación ecológica y económica como base para el ordenamiento territorial del departamento de Cajamarca. Cajamarca, Perú: Gobierno Regional de Cajamarca.

Hijmans, R. J., Cameron, S. E., Parra, J. L., Jones, P. G. y Jarvis, A. (2005). Very high resolution interpolated climate surfaces for global land areas. International Journal of Climatology, 25, 1965-1978.

INEI (Instituto Nacional de Estadística e Informática).(2007). Censos nacionales 2007: XI de población y VI de vivienda-Perú. Recuperado el 27 de julio de 2013 de: http://censos.inei.gob.pe/cpv2007/tabulados.

IUCN (International Union for Conservation of Nature).(2014). IUCN Red list of threatened species. Versión 2014.3. Recuperado el 18 de julio de 2014 de: www.iucnredlist.org/details/22066/0.

Killeen, T. J., Douglas, M., Consiglio, T., Jørgensen, P. M. y Mejía, J. (2007). Dry spots and wet spots in the Andean hotspot. Journal of Biogeography, $34,1357-1373$ 
Linares-Palomino, R. y Pennington, R. T. (2007). Lista anotada de plantas leñosas en bosques estacionalmente secos del Perú: una nueva herramienta en internet para estudios taxonómicos, ecológicos y de biodiversidad. Arnaldoa, 14, 149-152.

Lobo, J. M., Jiménez-Valverde, A. y Raimundo, R. (2008). AUC: a misleading measure of the performance of predictive distribution models. Global Ecology and Biogeography, 17, 145-151.

Minagri (Ministerio de Agricultura). (2014). Decreto supremo N . ${ }^{\circ}$ 004-2014Minagri: decreto supremo que aprueba la actualización de la lista de clasificación y categorización de las especies amenazadas de fauna silvestre legalmente protegidas. Diario Oficial El Peruano, 8 de abril de 2014. Lima Perú.

MTC (Ministerio de Transportes y Comunicaciones). (2013). Recuperado el 25 de noviembre de 2013 de: www.mtc.gob.pe/portal/transportes/red_vial/ mapas_redvial.htm.

Nazeri, M., Jusoff, K., Madani, N., Mahmud, A. R. y Bahman, A. R. (2012). Predictive modeling and mapping of Malayan sun bear (Helarctos malayanus) distribution using maximum entropy. PLoS ONE, 7, e48104. http://dx.doi.org/10.1371/journal.pone. 0048104

Olson, D. M., Dinerstein, E., Wikramanayake, E. D., Burgess, N. D., Powell, G. V. N., Underwood, E. C., et al. (2001). Terrestrial ecoregions of the world: a new map of life on Earth. Bioscience, 51, 933-938.

OY Ingeniería. (2009). Estudio de impacto ambiental del proyecto central hidroeléctrica Veracruz 730 mw. Resumen ejecutivo. Lima, Perú: Compañía Energética Veracruz, S.A.

Pearson, R. G. (2007). Species' distribution modeling for conservation educators and practitioners. American Museum of Natural History. Recuperado el 27 de julio de 2013 de: http://ncep.amnh.org.

Pearson, R. G., Raxworthy, C. J., Nakamura, M. y Peterson, A. T. (2007). Predicting species distributions from small numbers of occurrence records: a test case using cryptic geckos in Madagascar. Journal of Biogeography, 34, 102-117.

Peterson, A. T., Papeş, M. y Soberón, J. (2008). Rethinking receiver operating characteristic analysis applications in ecological niche modeling. Ecological Modelling, 213, 63-72.
Peyton, B. (1980). Ecology, distribution and food habits of spectacled bear, Tremarctos ornatus, in Peru. Journal of Mammalogy, 61, 639-652.

Phillips, S. J., Anderson, R. P. y Schapire, R. E. (2006). Maximum entropy modeling of species geographic distributions. Ecological Modelling, 190, 231-259.

Rodríguez, D., Cuesta, F., Goldstein, I., Bracho, A. E., Naranjo, L. G. y Hernández, O. L. (Eds.). (2003). Estrategia ecorregional para la conservación del oso andino en los Andes del Norte.. Bogotá: WWF Colombia, Fundación Wii, EcoCiencia, Wildlife Conservation Society.

Rodríguez-Achung, F. (2007). Manual para la zonificación ecológica y económica a nivel macro y meso. Iquitos, Perú: Instituto de Investigaciones de la Amazonía Peruana-IIAP, Proyecto Diversidad Biológica de la Amazonía Peruana-Biodamaz.

Roncal-Rabanal, M., Díaz-Mori, D., Roncal-Alcántara, C. y Rabanal-Díaz, W. (2013). Huacaybamba riqueza biológica del Marañón. Cajamarca, Perú: Universidad Nacional de Cajamarca.

Romo, M., Leo, M. y Epiquién, M. (2009). Propuesta de sistema de conservación regional-Sicre-Amazonas. Lima, Perú: Asociación Peruana para la Conservación de la Naturaleza.

Sánchez-Vega, I. y Sánchez-Rojas, A. (2012). La diversidad biológica en Cajamarca-Visión étnico-cultural y potencialidades. Cajamarca, Perú: Gobierno Regional Cajamarca.

Sernanp (Servicio Nacional de Áreas Naturales Protegidas por el Estado). (2014). Listado oficial de las áreas naturales protegidas. Recuperado el 11 de junio de 2014 de: www.sernanp.gob.pe/sernanp.

Vela, H. (2009). Meat consumption and use of parts of the Andean bear (Tremarctos ornatus) in the district of Chisquilla and Jumbilla, Bong County, Amazonas-Peru. International Bear News, 18, 20-21.

Wisz, M. S., Hijmans, R. J., Li, J., Peterson, A., Graham, C. H., Guisan, A., et al. (2008). Effects of sample size on the performance of species distribution models. Diversity and Distributions, 14, 763-773.

Yerena, E. (1993). El oso andino, especie clave para la conservación de la biodiversidad de la cordillera de los Andes. Flora, fauna y áreas silvestres. Venezuela. Flora, Fauna y Áreas Silvestres, 7, 32-37. 